\title{
Geometric Simulation Approach for Grading and Assessing the Thermostability of CALBs
}

\author{
B. Senthilkumar, D. Meshachpaul, and R. Rajasekaran \\ Computational Biology Lab, Department of Biotechnology, School of Bio Sciences and Technology, VIT University, \\ Vellore, Tamil Nadu 632014, India \\ Correspondence should be addressed to R. Rajasekaran; rrajasekaran@vit.ac.in
}

Received 30 November 2015; Accepted 16 March 2016

Academic Editor: Tzi Bun Ng

Copyright (C) 2016 B. Senthilkumar et al. This is an open access article distributed under the Creative Commons Attribution License, which permits unrestricted use, distribution, and reproduction in any medium, provided the original work is properly cited.

Candida antarctica lipase B (CALB) is a known stable and highly active enzyme used widely in biodiesel synthesis. In this work, the stability of native $(4 \mathrm{~K} 6 \mathrm{G})$ and mutant $(4 \mathrm{~K} 5 \mathrm{Q})$ CALB was studied through various structural parameters using conformational sampling approach. The contours of polar surface area and surface area of mutant CALB were $11357.67 \AA^{2}$ and $30007.4 \AA^{2}$, respectively, showing an enhanced stability compared to native CALB with a statistically significant $P$ value of $<0.0001$. Moreover, simulated thermal denaturation of CALB, a process involving dilution of hydrogen bond, significantly shielded against different intervals of energy application in mutant CALB revealing its augmentation of structural rigidity against native CALB. Finally, computational docking analysis showed an increase in the binding affinity of CALB and its substrate (triglyceride) in mutant CALB with Atomic Contact Energy (ACE) of $-91.23 \mathrm{kcal} / \mathrm{mol}$ compared to native CALB (ACE of $-70.3 \mathrm{kcal} / \mathrm{mol}$ ). The computational observations proposed that the use of mutant CALB (4K5Q) could serve as a best template for production of biodiesel in the future. Additionally, it can also be used as a template to identify efficient thermostable lipases through further mutations.

\section{Introduction}

Lipases (EC 3.1.1.3, triacylglycerol lipase) exist as proficient catalysts of high demand in varied fields. They are considered as remarkable molecular devices that determine patterns of chemical transformations like hydrolyzation, acidolysis, alcoholysis, aminolysis, esterification, interesterification, and transesterification [1]. Psychrophilic lipases are used widely in various industrial applications such as pharmaceutical productions, food industry, detergents, waste treatment, and bioremediation and recently in biodiesel production for their lipolytic activity. Microbes serve as a highly preferred source for wide variety of enzymes that are used in industrial firms. Industrial enzymes produced are widely adapted from yeast compared to bacteria, as yeast is easy to handle and grows in large scale [2].

Candida antarctica lipase B (CALB) obtained from the yeast Candida antarctica belongs to the enzyme class hydrolases, which specifically react with carboxylic ester bonds. Candida antarctica exhibits two forms of lipases (A and B) with varied physiochemical properties. Lipase B (CALB) is widely used in ester synthesis, desymmetrization, production of laboratory peracids, and other commercial products, as it is small, acidic, and less thermostable than lipase A [3]. CALB is an $\alpha / \beta$ globular protein with 317 amino acids weighing $33,273 \mathrm{Da}$, consisting of a conserved region with eight twisted $\beta$ strands and an $\alpha$-helix arranged on either side. CALB differs from other lipases as it is not shown to undergo interfacial activation, a phenomenon where lipase undergoes a conformational change associated with increased enzymatic activity when adsorbed onto a lipid surface $[1,4]$. CALB is a serine hydrolase, consisting of SerHis-Asp catalytic triad with serine acting as a nucleophile [5]. It is well known in industrial field for its chemical property involving the hydrolysis of triacylglycerols into fatty acids, diacylglycerol, monoacylglycerol, and glycerol [6]. It is characterized for its stereoselectivity, thermal stability, and activity, making it a common choice of enzyme for organic synthesis reactions and a competent biocatalyst for industrial applications. Industrial importance of CALB is due to its high enantioselectivity against secondary alcohols and its ability to survive high temperatures in organic solvents. Reduction 
in binding pocket size of CALB enables it to act highly on straight chain acids containing 5 to 12 carbon atoms [7].

CALB has advanced itself into the field of biodiesel production, as it possesses high stability and activity in the presence of alcohols. The industrial processing units involve high temperatures; hence, to cope with these challenges, improvement of a thermostable CALB with good enzyme activity is highly recommended. In this work, native CALB and mutant CALB were studied computationally using various structural stability features such as polar surface area (PSA), surface area (SA), energy distribution, hydrogen bond dilution, and docking to grade and assess their thermostability and functional activity. Experimental studies supported the reliability of CALB as a stable and active lipase but not much of computational works to support its stability are found. This study aims at computational prediction of thermostable CALB that could be used as a template for the synthesis of biodiesel and also an efficient model for appending further mutations to increase its strength in the future. In addition, the effect of conserved pattern in structural events using conformational sampling through normal mode-based geometric simulation showed reliable results as done by classical molecular dynamics comparatively [8]. Thus, this work aided in the computational understanding of CALB thermostable characterization and also instigating new development of efficient and robust computational method that could reduce time and laborious work.

\section{Materials and Methods}

2.1. Dataset. The steric constrained crystal structures of $4 \mathrm{~K} 6 \mathrm{G}$ (native) $\mathrm{CALB}$ and $4 \mathrm{~K} 5 \mathrm{Q}$ (mutant) CALB along with their related files were collected from Protein Data Bank (PDB) at http://www.rcsb.org/ [9]. The structural files were analyzed computationally for various physiochemical properties.

2.2. Structural Refinement and Conformational Sampling. The PDB structures of both native CALB and mutant CALB were structurally refined by KOBAMIN [10], which works on knowledge-based potential of mean force minimization, following which the structures were stereochemically corrected using MESHI. These energy minimized structures were scrutinized and utilized further in cluster analysis and single model analysis.

For cluster analysis, NMSim, a multiscale modeling program for protein conformational transitions, was used. Based on geometric simulations, the process was carried in three steps: (i) coarse grain of protein using FIRST software, (ii) normal mode analysis of rigid clusters, and (iii) geometric simulations (NMSim). Stereochemical ensemble of protein conformations along with their corresponding conformational trajectory and data plots were distinguished via structural analysis [11]. The calculations involving parameters such as polar surface area (PSA) and surface area (SA) of obtained conformational trajectories were evaluated via Vega ZZ, a molecular modeling program [12]. The variations in their values explained solvent accessibility and surface level interactions of the given protein.
The trajectory files of each protein were subjected to Bayesian Analysis Conformation Hunt (BACH), which discriminated the best model through BACHSCORE. The $\mathrm{BACH}$ program compares the given protein with similar protein structures obtained from various databases via global distant testing and atom energy scores. The program displayed protein conformations and their corresponding energy score as output; thus the one with least energy was predicted to be the best conformation (global minima) correspondingly [13]. This best conformation was used in single model analysis to compute effect of stability and functional activity by hydrogen bond dilution method and docking technique, respectively.

2.3. Hydrogen Bond Dilution. Various studies depicted the vital role played by hydrogen bonds in improved protein stability. The formation of stable hydrogen bonds relies on distance and angular distribution of both acceptor and donor molecules. On introduction of thermal energy, the hydrogen bonds begin to lose hold and break apart based on their distance, energy supplied, and their positions. MSU ProFlex (formerly called FIRST), a computational tool embedded in the program StoneHinge, was used to identify rigid and flexible regions of known proteins. StoneHingeP and StoneHingeD modules incorporated in StoneHinge program aided in the prediction of flexible hinge using ProFlex and its rigidity by DomDecomp analysis. Both analyses together helped in identifying both flexible and rigid regions of the given protein, CALB, in this study [14].

2.4. Docking Analysis. The role of receptor-ligand interaction helps to study the conceptual biology of protein function via docking analysis. A geometry-based molecular docking algorithm proposed by Schneidman-Duhovny et al. [15] was used for docking CALB with the substrate triglyceride. Triglyceride exists as a potent substrate for CALB both in vitro and in vivo. Hence, the SMILES string of molecule triglyceride was obtained from PubChem (http://pubchem.ncbi .nlm.nih.gov/) and its 3D structure was generated using CORINA (http://www.molecular-networks.com) [16]. Docking aids in identifying the transformation of one molecule at which other molecules could fit in devoid of any steric changes $[17,18]$. This depended not only on the geometric form of molecules but also on their complex stability. The program received both receptor and ligand molecules as PDB files, thus performing algorithmic calculations based on three stages: molecular shape representation, surface patch matching, and ranked scoring. The program rendered an output based on their shape complementarity, interface shape, and sizes of the molecules, and they were given as solutions along with their geometric score, desolvation energy, area size, and the docked PDB file. This helped to read the best template among mutants with high enzymatic activity [15]. The docked molecules were visualized using PyMOL, a molecular modeling package (The PyMOL Molecular Graphics System, Version 1.7.4 Schrödinger, LLC). 


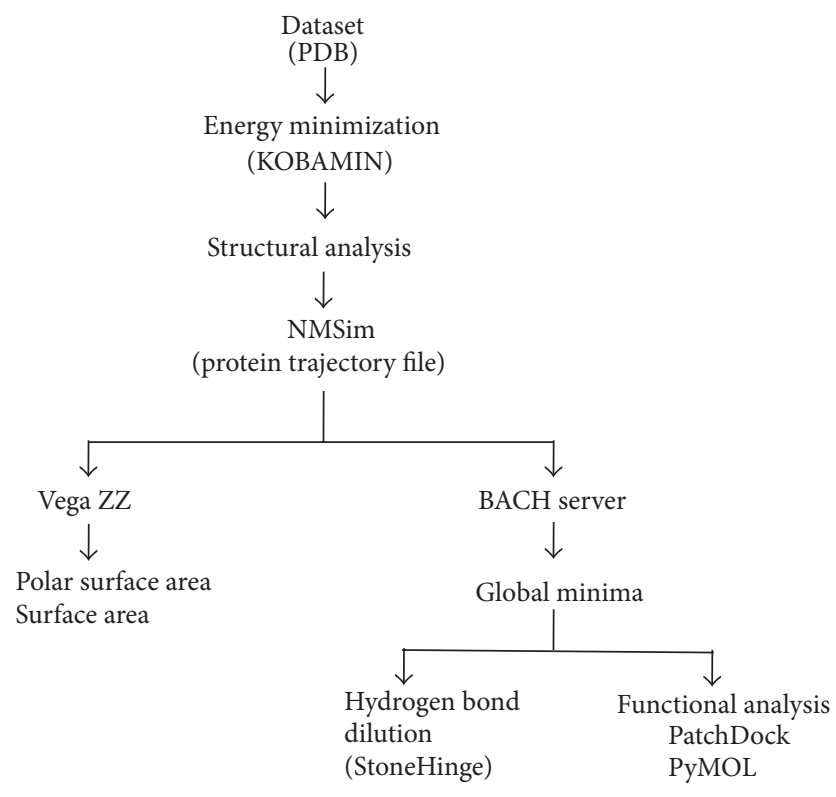

FIGURE 1: Flowchart illustrated the methodology and the various bioinformatics tools used in this study.

2.5. Statistical Analysis. The nonparametric values obtained via molecular simulation program were analyzed for statistical significance. Mann-Whitney test, also known as ranksum test, was utilized for the comparison of nonparametric unpaired groups [19]. Thereby, using MS-Excel, the nonparametric mean values of native CALB and mutant CALB were compared simultaneously. The significance of the parameters was calculated by two-tail probability value ( $P$ value) and $P<$ 0.05 was considered statistically significant.

\section{Results and Discussion}

Active site is said to play a vital role in enzymatic catalysis of a protein, thus aiding in its kinetic stability. The parameters constituting stability of native CALB (4K6G) and its most thermostatic mutant D223G/L278M (4K5Q) CALB protein [8] were evaluated based on the following: (a) polar surface area, (b) solvent accessibility, (c) energy distribution, (d) hydrogen bond dilution, and (e) binding affinity (Figure 1). These physiochemical parameters aided in the evaluation of structural stability and functionality of CALB, through conformational sampling.

\subsection{Retrieval of Native and Mutant Structure of CALB. CALB} belongs to $\alpha / \beta$ hydrolase class protein, globally known for its dual role in ester hydrolysis as well as in synthesis [4]. High resolution crystal structures $(<1.5 \AA)$ of native CALB and mutant CALB were collected from Protein Data Bank (PDB) for analyzing various physiochemical parameters contextual to thermostability. In this study, conformational sampling was utilized, which yielded an ensemble of protein conformations for cluster analysis. Subsequently, energetically best model among the conformations was selected for analyzing hydrogen bond dilution and binding affinity.
3.2. Degree of Solvability and Surface Accessibility of CALB. CALB exists as functionally known biocatalyst associated with the hydrolysis of water and esterification of various organic solvents [20]; hence, the study of interactions involving polar molecules in a solvable environment gains importance. CALB exhibited molecular heterogeneity, the ability of a molecule over time to assume several different conformations with trivial difference in stability, an innate property of all large biomolecules [21]. The cluster analysis was performed with the ensemble conformations obtained through the simulatory tool NMSim, using Vega ZZ program. The major parameters investigated for viewing the stability of a protein were RMSD and RMSF. Both these parameters showed high rigidity in mutant CALB compared to native CALB, similar to that of classic molecular dynamics studies [8], thereby demonstrating the eminence of structural variations in protein CALB stability and reliability of conformational sampling. With the reasoning of structural deviations for stability, stereochemical features of CALB at surface level, such as PSA and SA, were further analyzed.

The dissolution of a protein in water depends on two interactions: (1) the strength with which a protein molecule associates with the solvent and (2) how tightly the compound was bound to its own crystal lattice. PSA remains to be a part of van der Waals or solvent-accessible surface and their sum of surfaces that contained both polar $(\mathrm{O}, \mathrm{S}, \mathrm{N}, \mathrm{P}$, and $\mathrm{H}$ not bonded to $\mathrm{C}$ ) and apolar atoms ( $\mathrm{C}$ and $\mathrm{H}$ bonded to $\mathrm{C}$ ) in the given molecule. PSA was found to be increased along with the count of hydrogen bonds formed between protein surface and water, thereby constituting increased thermostability [22]. The PSA mean data of the mutant CALB $\left(11357.67 \AA^{2}\right)$ was found to be higher than that of the native CALB $\left(11328.08 \AA^{2}\right)$ with a statistically significant $P$ value (Mann-Whitney test, $P$ value $<0.0001$ ). Thus increased PSA was found to increase the hydrogen bonds relatively, thereby defining stability of the mutant CALB (4K5Q) as shown in Figure 2(a).

The ability of a protein molecule to interact with its solvent counterpart highly depends on the presence or absence of hydrophobic residues on its surface. As per previous studies, CALB was found capable of hydrolyzing soluble as well as insoluble substrates, with high level activity at water-lipid interfaces [23]. The ratio of buried nonpolar surface area to total nonpolar surface area of a protein was identified as hydrophobicity. Hydrophobic forces played a vital role in regard to thermostability and molecular folding of a protein $[24,25]$. Whereas the chemical reactions related to CALB majorly involved solvents in a solvable environment, exposure of polar residues on the surface was considered functionally important. The mean surface area of the mutant CALB $\left(30007.4 \AA^{2}\right)$ showed an increase compared to that of native CALB $\left(29862.5 \AA^{2}\right)$ with a statistically significant $P$ value less than 0.001 (Figure 2(b)). The results obtained might forecast the role polar residues played in sustaining functional aspect of mutant CALB, that is, activity in the presence of solvents such as alcohols.

3.3. Energy Allocation. The steric conformers simulated through NMSim were subjected for calculating energy 


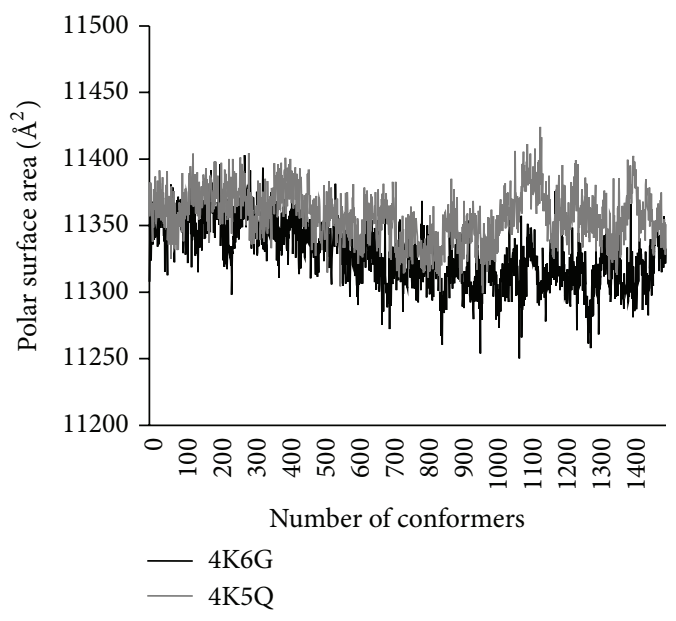

(a)

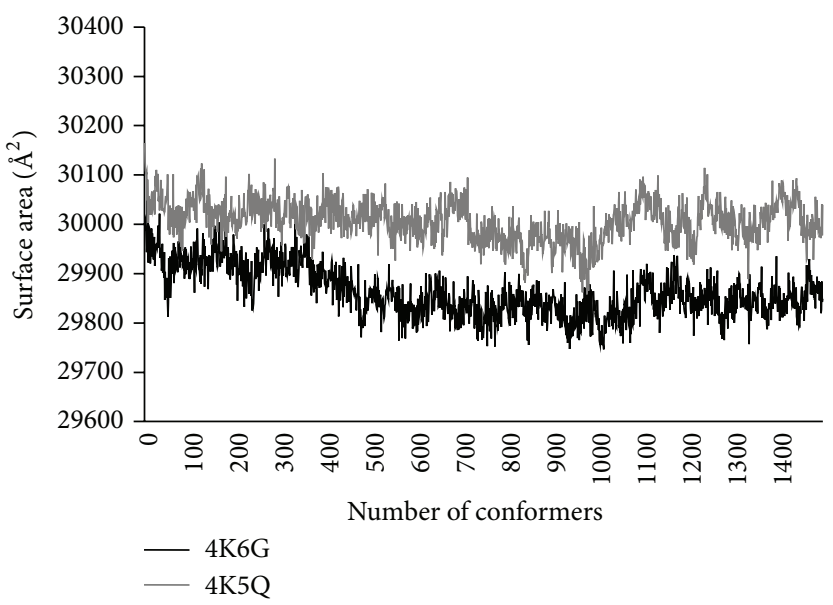

(b)

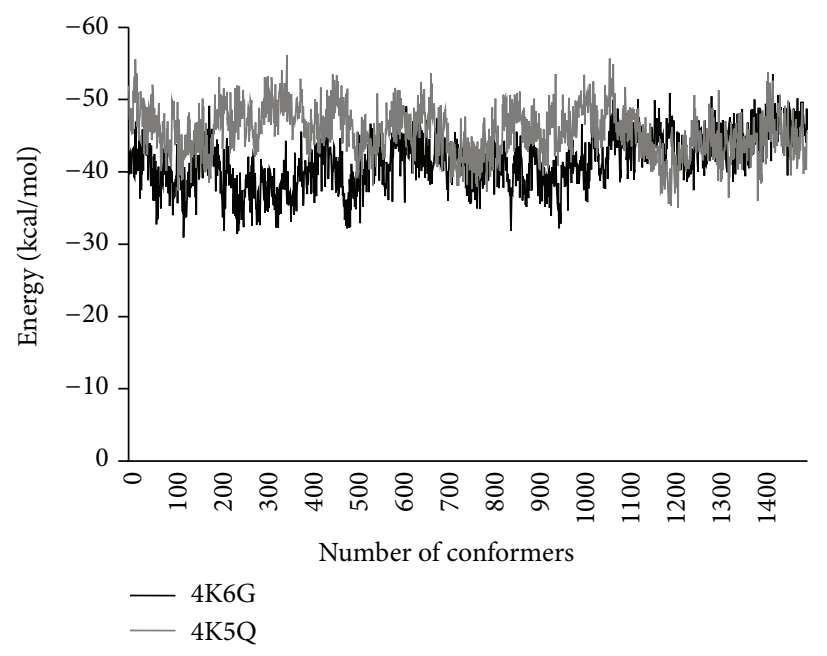

(c)

Figure 2: Geometrical observables of native (4K6G) CALB and mutant (4K5Q) CALB. (a) Polar surface area. (b) Surface area. (c) Energy levels calibrated by knowledge-based potential.

contour using $\mathrm{BACH}$ program. The BACH energy was calculated using effective pairwise residue-residue interactions along with single residue solvation property of the molecule. The graphical representation of energy profiles showed large number of mutant CALB conformers (mean energy: $-45.57 \mathrm{kcal} / \mathrm{mol}$ ) possessing low energy profiles compared to native CALB (mean energy: $-41.53 \mathrm{kcal} / \mathrm{mol}$ ) with statistically significant $P$ value less than 0.0001 , respectively (Figure 2(c)). Thereby a decrease in energy profile might be a corresponding factor for improved functionality and stability of mutant CALB compared to native CALB [13]. Among given conformations, the conformer with least energy also known as global minima was selected for further studies involving hydrogen bonds and functionality of CALB.

3.4. Hydrogen Bond Dilution of CALB. Among various stability enhancing interactions, hydrogen bonds are said to be an important parameter for stability. The global minima conformer of both native CALB and mutant CALB was selected to analyze hydrogen bond dilution, which may enlighten the understanding of mutant stability stereochemically. At intermittent state of energy levels, hydrogen bonds get diluted based on their steric properties, resulting in an unfolded protein structure, thus explaining the stability of a protein. The unfolding of protein structure resulted in loss of secondary structure and stability, due to breaking up of hydrogen bonds and salt bridges. Thermal denaturation was functionally implicated for the dilution of hydrogen bonds, thus resulting in breakage of weakest bonds followed by stronger ones. This in turn aided in the discovery of flexible and rigid areas of a given structural protein [14]. The hydrogen dilution pattern of native CALB and mutant CALB was analyzed using MSU ProFlex; the data obtained enriched us more of the role of hydrogen bonds in stability of CALB. Moreover, the results clearly stated that energy required to break a hydrogen bond was directly proportional to the strength of hydrogen bond; that is, the stronger the bonding the higher energy requirement $[26,27]$. The data 
TABLE 1: Hydrogen bond dilution data of native (4K6G) and mutant (4K5Q) CALB showing the number of bonds broken and the number of bonds remaining to be broken at different energy profile.

\begin{tabular}{|c|c|c|c|c|c|}
\hline \multirow{2}{*}{ SI number } & \multirow{2}{*}{ Energy supplied (kcal/mol) } & \multicolumn{2}{|c|}{$4 \mathrm{~K} 6 \mathrm{G}$} & \multicolumn{2}{|c|}{ 4K5Q } \\
\hline & & Bonds broken & Bonds remaining & Bonds broken & Bonds remaining \\
\hline 1 & $<-0.9$ & 36 & 233 & 41 & 237 \\
\hline 2 & $-1<-1.9$ & 34 & 199 & 41 & 196 \\
\hline 3 & $-2<-2.9$ & 32 & 167 & 49 & 147 \\
\hline 4 & $-3<-3.9$ & 39 & 128 & 28 & 119 \\
\hline 5 & $-4<-4.9$ & 37 & 91 & 32 & 87 \\
\hline 6 & $-5<-5.9$ & 40 & 51 & 45 & 42 \\
\hline 7 & $-6<-6.9$ & 34 & 17 & 23 & 19 \\
\hline 8 & $-7<-7.9$ & 0 & 0 & 16 & 3 \\
\hline
\end{tabular}

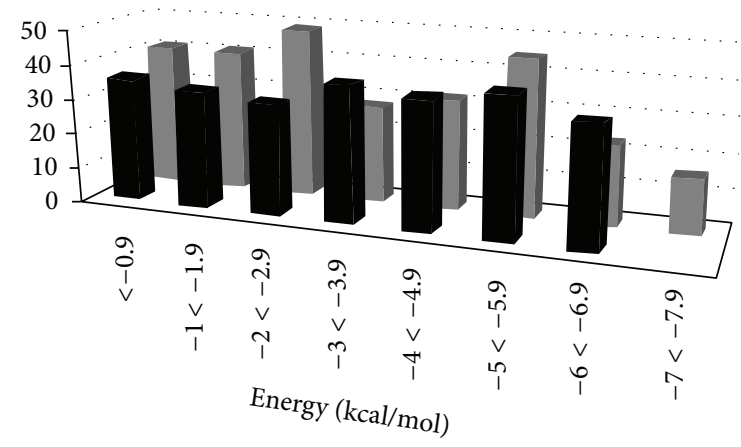

$-4 \mathrm{~K} 6 \mathrm{G}$

$$
-4 \mathrm{~K} 5 \mathrm{Q}
$$

(a)

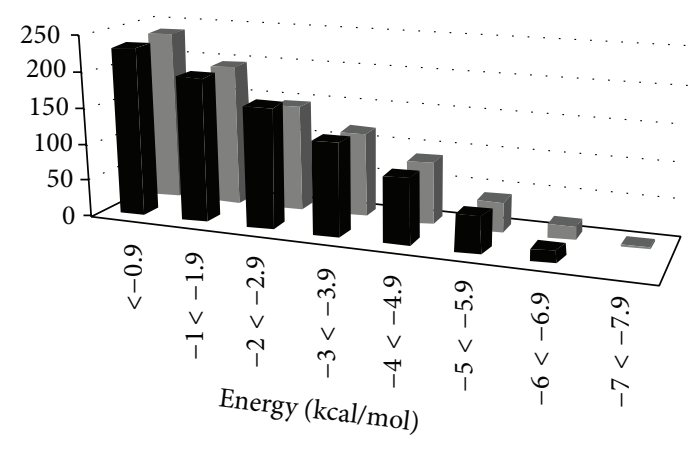

$-4 \mathrm{~K} 6 \mathrm{G}$

(b)

FIGURE 3: Comparative graphical representation of (a) the number of hydrogen bonds broken and (b) the number of hydrogen bonds remaining to be broken, at different energy levels for native (4K6G) CALB and mutant (4K5Q) CALB.

depicting the number of bonds broken after each energy cycle (Table 1) predicted the strength and steric stability of remaining hydrogen bonds in maintaining overall structural flexibility and rigidity of CALB.

Three stable hydrogen bonds remained unbroken in mutant CALB after supplying energy of more than $-8 \mathrm{kcal} / \mathrm{mol}$. In contrast, complete hydrogen bond dilution took place before supplying energy more than $-7 \mathrm{kcal} / \mathrm{mol}$ in native CALB (Figure 3). The data also showed that a significant number of bonds were broken both in native CALB (40) and in mutant CALB (45) between energy levels of $-5 \mathrm{kcal} / \mathrm{mol}$ and $-5.9 \mathrm{kcal} / \mathrm{mol}$ comparatively. In addition to current observations earlier studies too supported the fact that hydrogen bonding increases stability and rigidity of CALB [22].

3.5. Binding Affinity of $C A L B$. The functional enzymatic efficiency and binding capacity of both native CALB and mutant CALB with their substrate triglyceride were studied using PatchDock. The program expressed the results as Atomic Contact Energy (ACE). The ACE of the mutant CALB $(-91.23 \mathrm{kcal} / \mathrm{mol})$ showed high binding affinity compared to that of native CALB $(-70.3 \mathrm{kcal} / \mathrm{mol})$ at active site 130 [25]. The result showed a change induced in native CALB due to mutation could be a potent reason for the increase in binding efficiency of its mutant counterpart. The alteration in symmetric conformation of superimposed docked complexes of both native CALB and mutant CALB was illustrated in Figure 4, representing the point at which CALB was appended with substrate triglyceride. The ligand molecules were bound in such a manner that they face each other at a single point.

\section{Conclusions}

CALB exists as highly industrial oriented enzyme in present age and is known for its various functional aspects predominantly in biodiesel production. The native CALB and its corresponding mutant CALB were analyzed computationally; as biodiesel production involves hydrolysis of alcohols by CALB, an in-depth analysis of parameters like solubility and surface accessibility gains importance in this study. Cluster analysis was carried out using conformation sampling method in which PSA and SA of mutant CALB showed their significance in solvent accessibility, thereby defining functional activity of CALB. Hydrogen bond dilution showed lack of stable hydrogen bonds in native CALB beyond energy level of $-7 \mathrm{kcal} / \mathrm{mol}$, whereas mutant CALB showed a 


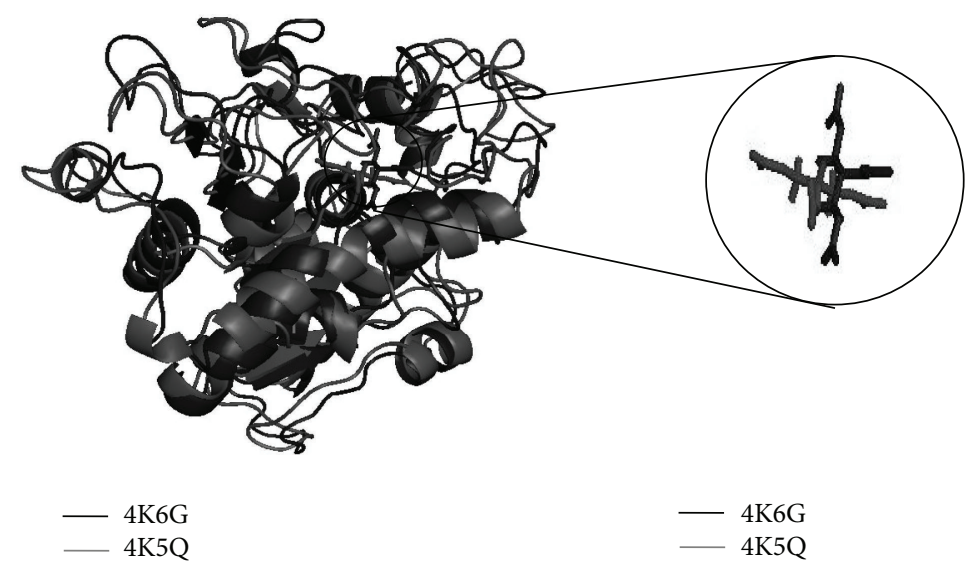

(a)

(b)

Figure 4: An illustration of the docked enzymes native CALB (4K6G) and mutant CALB (4K5Q) bound to their ligand triglyceride: (a) superimposed structure of the docked CALB and (b) the orientation of the ligand triglyceride at the active site 130.

presence of 22 bonds to be thermally denatured at a supply of more than $-7 \mathrm{kcal} / \mathrm{mol}$ of energy. In addition, docking analysis performed using PatchDock showed a difference in ACE between the mutant CALB $(-91.23 \mathrm{kcal} / \mathrm{mol})$ and native CALB $(-70.3 \mathrm{kcal} / \mathrm{mol})$, representing their functional enzymatic activity, which depicted an increase in mutant binding affinity. The characterization and production of thermostable lipase with selective activity became a great demand in the field of biotechnology [28]. This study clearly states that the use of CALB mutant as a stable template could be a reliable source for enzymatic processes in biotechnology firm. It could also be utilized as a starter template for introducing mutations and designing high thermostable CALBs in the future. Moreover, the application of conformational sampling showed a lucid level of similarity with classic molecular dynamics, thus supporting the use of conformational sampling in simulation analysis aiding in timely results.

\section{Competing Interests}

The authors declare that they have no competing interests.

\section{Acknowledgments}

The authors thank the management of VIT University for providing the facilities and encouragement to carry out this research work.

\section{References}

[1] M. R. Ganjalikhany, B. Ranjbar, A. H. Taghavi, and T. Tohidi Moghadam, "Functional motions of candida antarctica lipase b: a survey through open-close conformations," PLoS ONE, vol. 7, no. 7, Article ID e40327, 2012.

[2] J. Vakhlu and A. Kour, "Yeast lipases: enzyme purification, biochemical properties and gene cloning," Electronic Journal of Biotechnology, vol. 9, no. 1, pp. 69-85, 2006.
[3] K. S. Siddiqui and R. Cavicchioli, "Improved thermal stability and activity in the cold-adapted lipase B from Candida antarctica following chemical modification with oxidized polysaccharides," Extremophiles, vol. 9, no. 6, pp. 471-476, 2005.

[4] A. Idris and A. Bukhari, "Immobilized candida antarctica lipase B: hydration, stripping off and application in ring opening polyester synthesis," Biotechnology Advances, vol. 30, no. 3, pp. 550-563, 2012.

[5] M. Irani, U. Törnvall, S. Genheden, M. W. Larsen, R. Hatti-Kaul, and U. Ryde, "Amino acid oxidation of Candida antarctica lipase $B$ studied by molecular dynamics simulations and site-directed mutagenesis," Biochemistry, vol. 52, no. 7, pp. 1280-1289, 2013.

[6] A. Houde, A. Kademi, and D. Leblanc, "Lipases and their industrial applications: an overview," Applied Biochemistry and Biotechnology - Part A: Enzyme Engineering and Biotechnology, vol. 118, no. 1-3, pp. 155-170, 2004.

[7] J. Pfeffer, S. Richter, J. Nieveler et al., "High yield expression of Lipase A from Candida antarctica in the methylotrophic yeast Pichia pastoris and its purification and characterisation," Applied Microbiology and Biotechnology, vol. 72, no. 5, pp. 931938, 2006.

[8] Y. Xie, J. An, G. Yang et al., "Enhanced enzyme kinetic stability by increasing rigidity within the active site," Journal of Biological Chemistry, vol. 289, no. 11, pp. 7994-8006, 2014.

[9] H. M. Berman, J. Westbrook, Z. Feng et al., "The protein data bank," Nucleic Acids Research, vol. 28, no. 1, pp. 235-242, 2000.

[10] J. P. G. L. M. Rodrigues, M. Levitt, and G. Chopra, "KoBaMIN: a knowledge-based minimization web server for protein structure refinement," Nucleic Acids Research, vol. 40, no. 1, pp. W323-W328, 2012.

[11] D. M. Krüger, A. Ahmed, and H. Gohlke, "NMSim web server: integrated approach for normal mode-based geometric simulations of biologically relevant conformational transitions in proteins," Nucleic Acids Research, vol. 40, no. W1, pp. W310W316, 2012.

[12] A. Pedretti, L. Villa, and G. Vistoli, "VEGA: a versatile program to convert, handle and visualize molecular structure on Windows-based PCs," Journal of Molecular Graphics \& Modelling, vol. 21, no. 1, pp. 47-49, 2002. 
[13] P. Cossio, D. Granata, A. Laio, F. Seno, and A. Trovato, "A simple and efficient statistical potential for scoring ensembles of protein structures," Scientific Reports, vol. 2, article 351, 2012.

[14] K. S. Keating, S. C. Flores, M. B. Gerstein, and L. A. Kuhn, "StoneHinge: hinge prediction by network analysis of individual protein structures," Protein Science, vol. 18, no. 2, pp. 359-371, 2009.

[15] D. Schneidman-Duhovny, Y. Inbar, R. Nussinov, and H. J. Wolfson, "PatchDock and SymmDock: servers for rigid and symmetric docking," Nucleic Acids Research, vol. 33, no. 2, pp. W363-W367, 2005.

[16] J. Sadowski, J. Gasteiger, and G. Klebe, "Comparison of automatic three-dimensional model builders using $639 \mathrm{X}$-ray structures," Journal of Chemical Information and Computer Sciences, vol. 34, no. 4, pp. 1000-1008, 1994.

[17] Y.-C. Lou, I. Wang, M. Rajasekaran et al., "Solution structure and tandem DNA recognition of the C-terminal effector domain of PmrA from Klebsiella pneumoniae," Nucleic Acids Research, vol. 42, no. 6, pp. 4080-4093, 2014.

[18] C.-C. Chou, M. Rajasekaran, and C. Chen, "An effective approach for generating a three-Cys2His2 zinc-finger-DNA complex model by docking," BMC Bioinformatics, vol. 11, article 334, 2010.

[19] A. Kundu and W.-R. Wu, "Wilcoxon-Mann-Whitney rank testbased filter for removing streak noise in transmitted DPCM images," IEEE Transactions on Communications, vol. 38, no. 2, pp. 150-155, 1990.

[20] H. J. Park, J. C. Joo, K. Park, Y. H. Kim, and Y. J. Yoo, "Prediction of the solvent affecting site and the computational design of stable Candida antarctica lipase B in a hydrophilic organic solvent," Journal of Biotechnology, vol. 163, no. 3, pp. 346-352, 2013.

[21] P. Cysewski, "Apparent basicities of the surfaces characterizing the dominant crystal habits of distinct polymorphic forms of 4aminosulfonamide," Journal of Molecular Modeling, vol. 20, no. 7, article 2276, 2014.

[22] G. Vogt, S. Woell, and P. Argos, "Protein thermal stability, hydrogen bonds, and ion pairs," Journal of Molecular Biology, vol. 269, no. 4, pp. 631-643, 1997.

[23] C. C. Gruber and J. Pleiss, "Lipase B from Candida antarctica binds to hydrophobic substrate-water interfaces via hydrophobic anchors surrounding the active site entrance," Journal of Molecular Catalysis B: Enzymatic, vol. 84, pp. 48-54, 2012.

[24] C. N. Pace, H. Fu, K. L. Fryar et al., "Contribution of hydrophobic interactions to protein stability," Journal of Molecular Biology, vol. 408, no. 3, pp. 514-528, 2011.

[25] J. Uppenberg, N. Öhrner, M. Norin et al., "Crystallographic and molecular-modeling studies of lipase B from Candida antarctica reveal a stereospecificity pocket for secondary alcohols," Biochemistry, vol. 34, no. 51, pp. 16838-16851, 1995.

[26] R. Mahalingam, H.-P. Peng, and A.-S. Yang, "Prediction of fatty acid-binding residues on protein surfaces with threedimensional probability distributions of interacting atoms," Biophysical Chemistry, vol. 192, pp. 10-19, 2014.

[27] R. Mahalingam, H.-P. Peng, and A.-S. Yang, "Prediction of FMN-binding residues with three-dimensional probability distributions of interacting atoms on protein surfaces," Journal of Theoretical Biology, vol. 343, pp. 154-161, 2014.

[28] K.-E. Jaeger and M. T. Reetz, "Microbial lipases form versatile tools for biotechnology," Trends in Biotechnology, vol. 16, no. 9, pp. 396-403, 1998. 

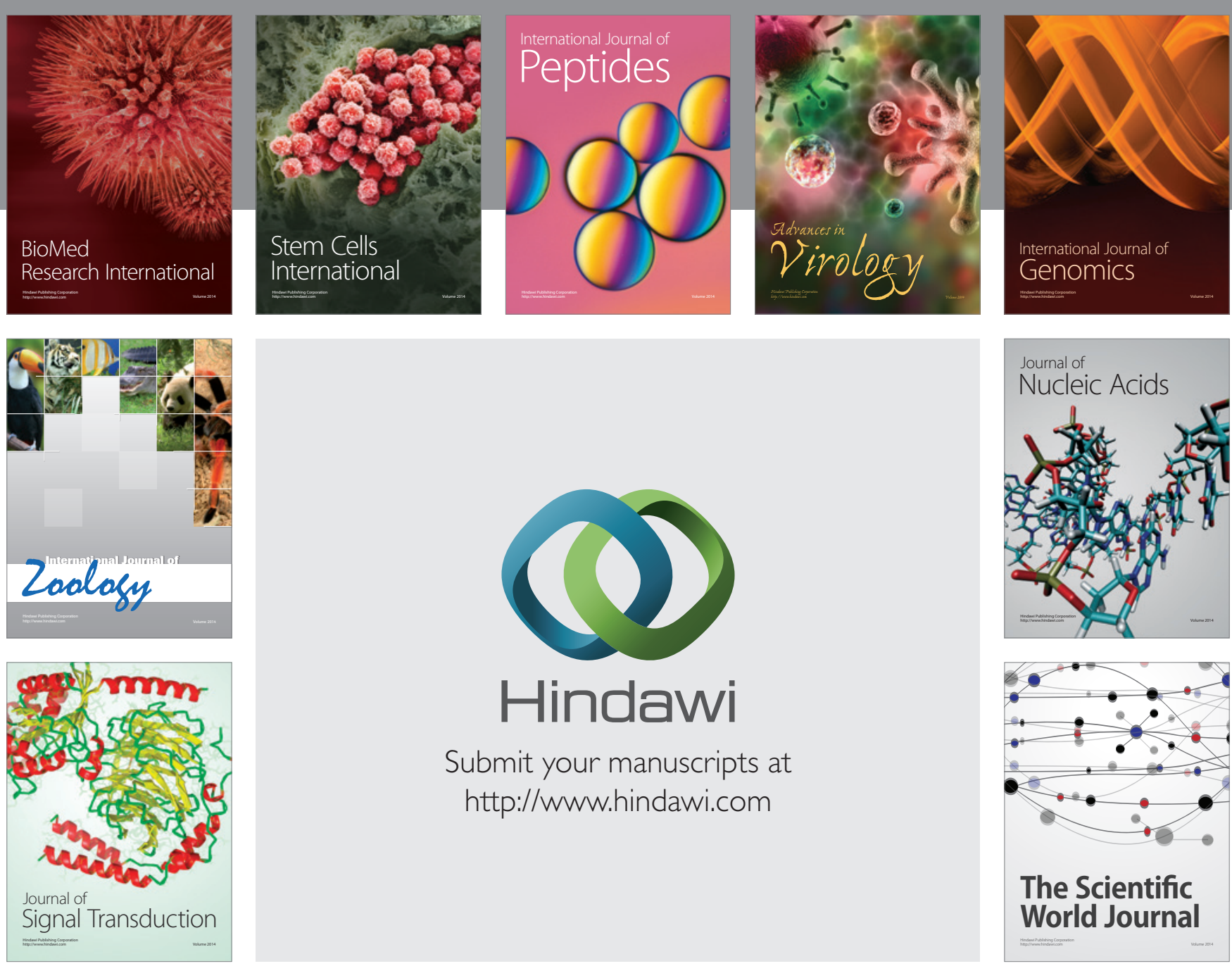

Submit your manuscripts at

http://www.hindawi.com
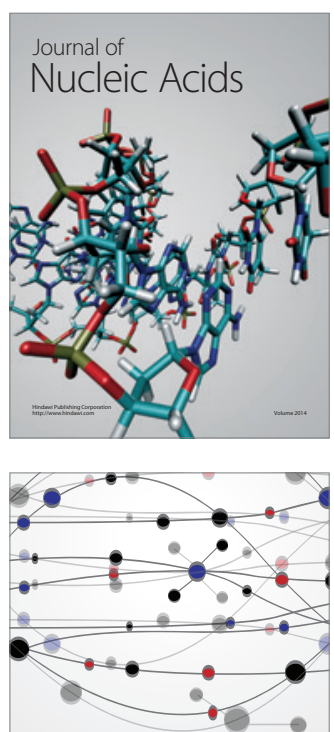

The Scientific World Journal
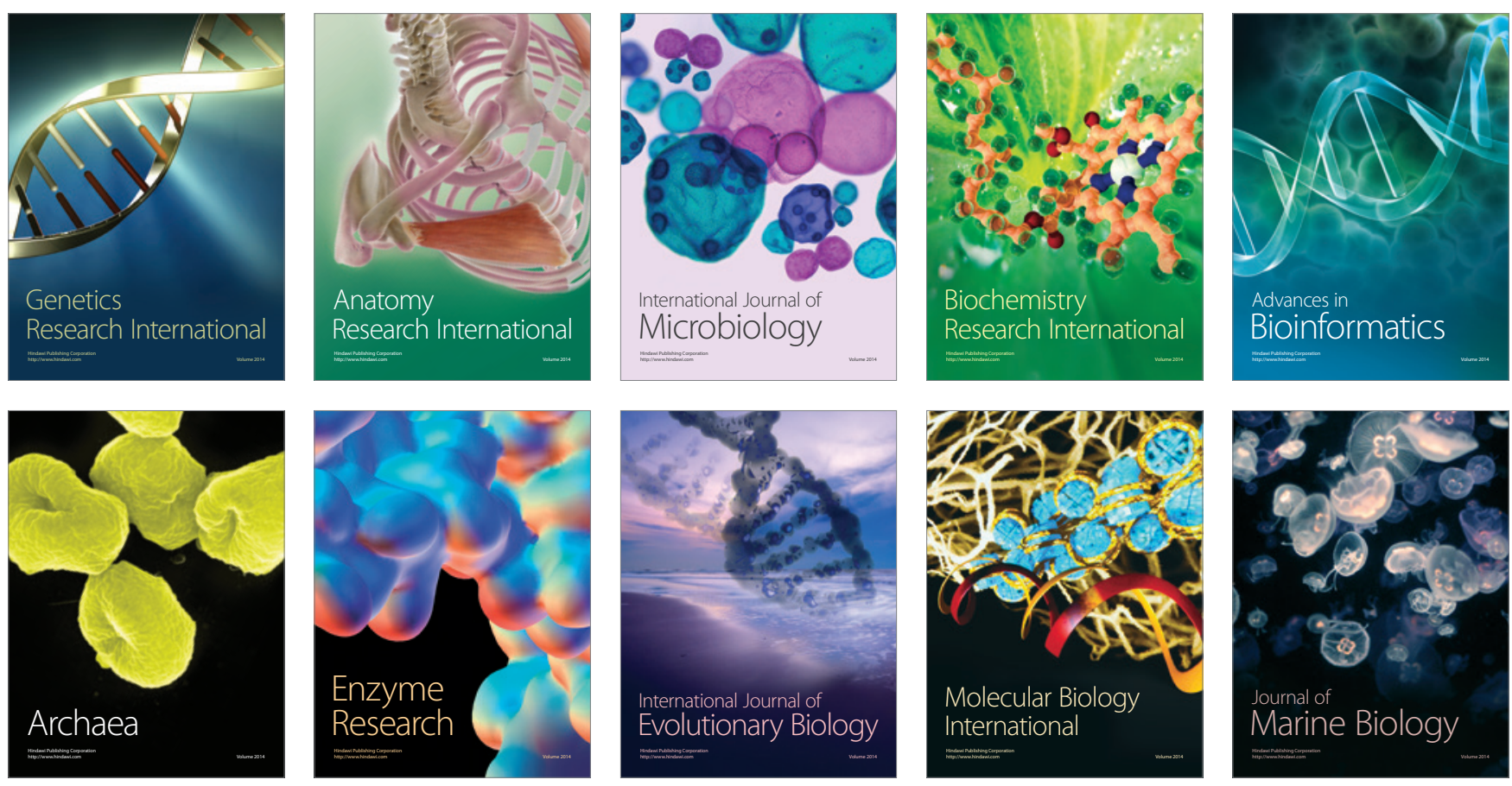